\title{
A Generalized Power Lindley Distribution: Model, Properties and Applications
}

\author{
Hamzeh Torabi \\ Yazd University, Islamic Republic of Iran \\ htorabi@yazd.ac.ir \\ Mojgan Baghaeipoor \\ Islamic Republic of Iran \\ m.baghaeepoor@gmail.com \\ Narges Montazeri \\ Islamic Republic of Iran \\ nmontazeri@stu.yazd.ac.ir \\ G.G. Hamedani \\ Marquette University, USA \\ gholamhoss.hamedani@marquette.edu
}

\begin{abstract}
In this paper, a three parameters generalization of the power Lindley distribution is introduced. This includes as special cases the power Lindley and Lindley distribution. The new distribution exhibits decreasing, increasing and bathtub hazard rate depending on its parameters. Several statistical properties of the distribution are explored. Then, a bivariate version of the proposed distribution is derived. Finally, three real data applications illustrate the performance of our proposed distribution.
\end{abstract}

Keywords: Characterizations; Generalized; Power; Lindley distribution; Stacy gamma distribution; Hazard rate function; Stochastic order; Mean resudual life; AIC; BIC; CAIC; HQIC.

Mathematics Subject Classification (2010): 60E12, 62A10.

\section{Introduction}

The Lindley distribution with probability density function (pdf)

$$
f(x ; \beta)=\frac{\beta^{2}(1+x) e^{-x \beta}}{1+\beta}, \quad x>0, \quad \beta>0,
$$

was introduced by Lindley [(1958)] to illustrate a difference between fiducial distribution and posterior distribution. Ghitany et al. [(2013)] introduced a family of distributions with the pdf

$$
f(x ; \alpha, \beta)=\frac{\alpha \beta^{2}}{\beta+1}\left(1+x^{\alpha}\right) x^{\alpha-1} e^{-\beta x^{\alpha}}, \quad x>0, \quad \beta, \theta>0,
$$

A random variable with pdf (2) is said to have the power Lindley (PL) distribution. This article offers a distribution which generalizes the power Lindley distribution is based on certain mixture of two Stacy gamma distributions. The study discusses various properties of the new model. 
The paper is organized as follows: Section 2 introduces a generalized power Lindley (GPL) distribution and presents its basic properties including the behaviour of the density and hazard rate functions, and some results on stochastic orderings. The moments of GPL distribution and its characteristic function are derived in Section 3. Mean residual function is obtained in Section 4. The Lorenz curve and Bonferroni curve are obtained in Section 5. Section 6 presents certain characterizations of GPL distribution and then the estimation of parameters is discussed in Section 7. We also proposed an algorithm for generating random data from the new distribution in Section 8. In Section 9, we present the simulation issues of the GPL distribution. Some applications of the GPL distribution and comparison with other distributions, are given in Section 10.

\section{Definition and some properties of GPL}

In this section, we introduce a GPL distribution and study its basic properties. Assume

$$
f_{g g}(x ; \alpha, \beta, \gamma)=\frac{\alpha \beta^{\frac{\gamma}{\alpha}} e^{-\beta \mathrm{x}} x^{\gamma-1}}{\Gamma\left[\frac{\gamma}{\alpha}\right]}, \quad x>0, \quad \alpha, \beta, \gamma>0,
$$

is the density function of the generalized gamma (Stacy gamma) distribution, denoted by $\mathrm{GG}(\alpha, \beta, \gamma)$.

Let $f_{1}(x ; \alpha, \beta, \gamma)$ and $f_{2}(x ; \alpha, \beta, 2 \gamma)$ be pdf's of $\operatorname{GG}(\alpha, \beta, \gamma)$ and $\operatorname{GG}(\alpha, \beta, 2 \gamma)$ respectively. Let $X$ be a random variable with pdf given by $f(x ; \alpha, \beta, \gamma)=\frac{\beta}{1+\beta} f_{1}(x ; \alpha, \beta, \gamma)+\frac{1}{1+\beta} f_{2}(x ; \alpha, \beta, 2 \gamma)$, i.e., $f(x ; \alpha, \beta, \gamma)$ is a mixture pdf. Then the pdf of $X$ is as follows:

$$
f(\mathrm{x}, \alpha, \beta, \gamma)=\frac{e^{-x^{\alpha} \beta} x^{-1+\gamma} \alpha \beta^{\frac{\gamma}{\alpha}}\left(x^{\gamma} \beta^{\frac{\gamma}{\alpha}} \Gamma\left[\frac{\gamma}{\alpha}\right]+\beta \Gamma\left[\frac{2 \gamma}{\alpha}\right]\right)}{(1+\beta) \Gamma\left[\frac{\gamma}{\alpha}\right] \Gamma\left[\frac{2 \gamma}{\alpha}\right]}, x>0, \alpha, \beta, \gamma>0 .
$$

We say that the random variable $X$ has a GPL distribution, if $X$ has the density function defined by (4) and use the notation $\operatorname{GPL}(\alpha, \beta, \gamma)$.

\subsection{Special cases of the GPL distribution}

The GPL distribution has a number of distributions as special cases as follows:

(a) For $\gamma=\alpha$, the GPL distribution reduces to PL distribution (2) with parameters $\alpha$ and $\beta$;

(b) For $\alpha=\gamma=1$, GPL distribution reduces to of the Lindley distribution (1) with parameters $\beta$. 


\subsection{Shape}

In this section, we discuss the shape characteristics of pdf (4). The behavior of its pdf at $x=0$ and at $\infty$ are as follows:

$$
\lim _{x \rightarrow 0} f(x ; \alpha, \beta, \gamma)=\left\{\begin{array}{ll}
\infty, & \text { if } \gamma<1, \\
0, & \text { if } \gamma>1,
\end{array} \lim _{x \rightarrow \infty} f(x ; \alpha, \beta, \gamma)=0 .\right.
$$

Also we have

$$
\rho(x):=\frac{d}{d x} \log f(x)=-\frac{1+x^{\alpha} \alpha \beta-2 \gamma+\frac{\beta \gamma \Gamma\left[\frac{2 \gamma}{\alpha}\right]}{x^{\gamma} \beta^{\frac{\gamma}{\alpha}} \Gamma\left[\frac{\gamma}{\alpha}\right]+\beta \Gamma\left[\frac{2 \gamma}{\alpha}\right]}}{x},
$$

and

$$
\frac{d^{2}}{d x^{2}} \log f(x)=\frac{1-x^{\alpha}(-1+\alpha) \alpha \beta-2 \gamma}{x^{2}}+\frac{\beta \Gamma\left[\frac{2 \gamma}{\alpha}\right]\left(x^{\gamma} \beta^{\frac{\gamma}{\alpha}} \gamma(1+\gamma) \Gamma\left[\frac{\gamma}{\alpha}\right]+\beta \gamma\left[\frac{2 \gamma}{\alpha}\right]\right)}{x^{2}\left(x^{\gamma} \beta^{\frac{\gamma}{\alpha}} \Gamma\left[\frac{\gamma}{\alpha}\right]+\beta \Gamma\left[\frac{2 \gamma}{\alpha}\right]\right)^{2}} .
$$

It is clear that if $\alpha=1$ and $\gamma, \leq \frac{1}{2}$, then $\frac{d^{2}}{d x^{2}} \log f(x) \geq 0$; i.e., the pdf is log-concave and hence unimodal.

Figure 1 Shows plots of the pdf of the GPL distribution for some selected parameters $\alpha$, $\beta$ and $\gamma$.

Figure 1: Plots of the pdf of the GPL distribution for some selected parameters $\alpha, \beta$ and $\gamma$.
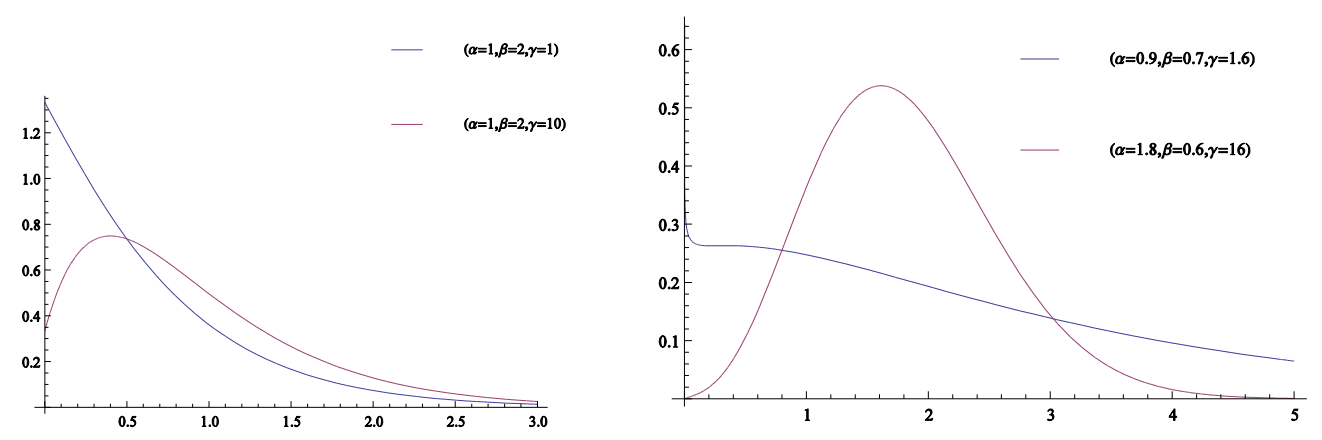

The hazard rate function of the random variable $X$ distributed according to $\operatorname{GPL}(\alpha, \beta, \gamma)$ is 


$$
h(x)=\frac{f(x)}{1-F(x)}=\frac{e^{-x^{\alpha} \beta} x^{-1+\gamma} \alpha \beta^{\frac{\gamma}{\alpha}}\left(x^{\gamma} \beta^{\frac{\gamma}{\alpha}} \Gamma\left[\frac{\gamma}{\alpha}\right]+\beta \Gamma\left[\frac{2 \gamma}{\alpha}\right]\right)}{\beta \Gamma\left[\frac{2 \gamma}{\alpha}\right] \Gamma\left[\frac{\gamma}{\alpha}, x^{\alpha} \beta\right]+\Gamma\left[\frac{\gamma}{\alpha}\right] \Gamma\left[\frac{2 \gamma}{\alpha}, x^{\alpha} \beta\right]} .
$$

Then $h(x)$ has different behaviours depending on its parameters. Lemma 2.1 shows that the distribution can have increasing hazard rate (IFR) for a special case.

Lemma 2.1 Let $h(x)$ be the hazard function of a random variable $X$ distributed according to the $G P L(\alpha, \beta, \gamma)$. Then $h(x)$ is increasing for $\alpha=1, \gamma \leq \frac{1}{2}$;

Proof. From (5), we have

$$
\rho^{\prime}(x)=\frac{1-x^{\alpha}(-1+\alpha) \alpha \beta-2 \gamma}{x^{2}}+\frac{\beta \Gamma\left[\frac{2 \gamma}{\alpha}\right]\left(x^{\gamma} \beta^{\frac{\gamma}{\alpha}} \gamma(1+\gamma) \Gamma\left[\frac{\gamma}{\alpha}\right]+\beta \gamma \Gamma\left[\frac{2 \gamma}{\alpha}\right]\right)}{x^{2}\left(x^{\gamma} \beta^{\frac{\gamma}{\alpha}} \Gamma\left[\frac{\gamma}{\alpha}\right]+\beta \Gamma\left[\frac{2 \gamma}{\alpha}\right]\right)^{2}} .
$$

It follows that $\rho^{\prime}(x) \geq 0$ when $\alpha=1$ and $\gamma \leq, \frac{1}{2}$. This completes the proof of the lemma.

Figure 2 Shows the hazard rate function of the GPL distribution for some selected parameters $\alpha, \beta$ and $\gamma$.

Figure 2: Plots of the hazard function of the GPL distribution for some selected parameters $\alpha, \beta$ and $\gamma$.
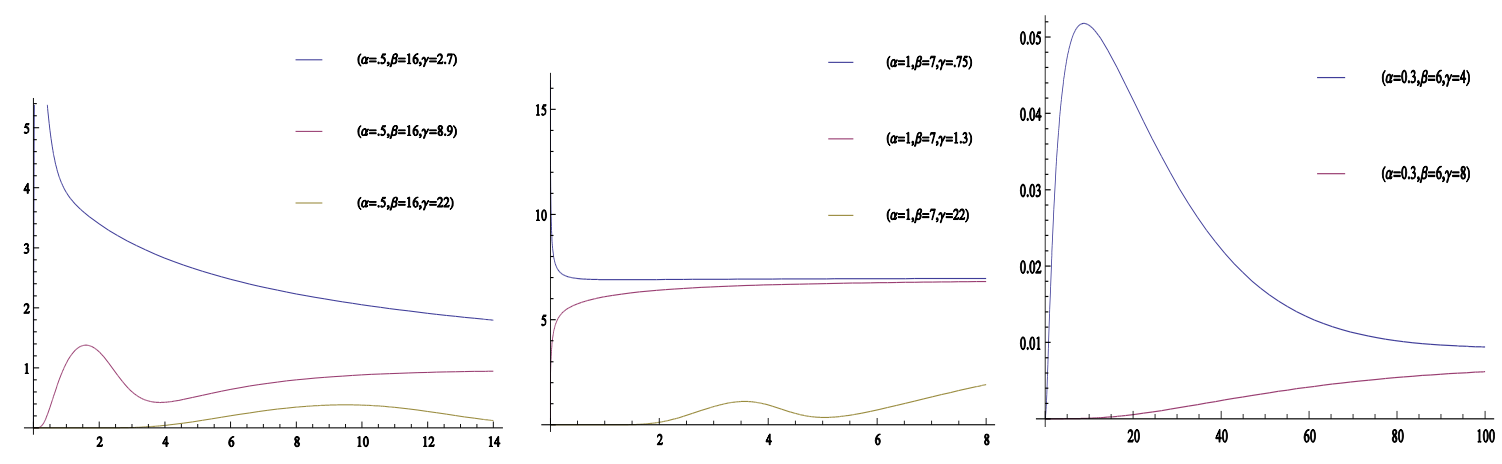

\subsection{Stochastic Orders}

A random variable $X$ is said to be stochastically smaller than $Y$, denoted by $X \pi_{s} Y$, if $F_{X}(t) \geq F_{Y}(t)$ for all $t$. 
A generalized power Lindley distribution: Model, Properties and Applications.

Two stronger criteria are the hazard rate order denoted by $\mathrm{X} \pi_{h r} \mathrm{Y}$ if $h_{X}(t) \geq h_{Y}(t)$, for all $t$, and the likelihood ratio order denoted by $X \pi_{l r} Y$, if $f_{X}(t) / f_{Y}(t)$ is decreasing in $t$. Note that

$$
X \pi_{l r} Y \Rightarrow X \pi_{h r} Y \Rightarrow X \pi_{s} Y \text {. }
$$

For details of the proof, see Shaked and Shanthikumar [(1994)].

Let $X_{i}$ be a random variable with pdf (4) and parameters $\left(\alpha_{i}, \beta_{i}, \gamma_{i}\right)$, for $i=1,2$. Then

$$
\begin{aligned}
& \frac{d}{d x} \log \left(\frac{f_{X_{1}}(x)}{f_{X_{2}}(x)}\right)=\frac{d}{d x}\left[\log f_{X_{1}}(x)-\log f_{X_{2}}(x)\right] \\
& =x^{-1}\left(\frac{\beta_{2}^{\frac{\gamma_{2}}{\alpha_{2}}} x^{\gamma_{2}}\left(-2 \gamma_{2}+\alpha_{2} \beta_{2} x^{\alpha_{2}}-x^{\alpha_{1}} \alpha_{1} \beta_{1}+2 \gamma_{1}\right) \Gamma\left[\frac{\gamma_{2}}{\alpha_{2}}\right]}{\beta_{2}^{\frac{\gamma_{2}}{\alpha_{2}}} x^{\gamma_{2}} \Gamma\left[\frac{\gamma_{2}}{\alpha_{2}}\right]+\beta_{2} \Gamma\left[\frac{2 \gamma_{2}}{\alpha_{2}}\right]}\right. \\
& +\frac{\beta_{2}\left(-\gamma_{2}+\alpha_{2} \beta_{2} x^{\alpha_{2}}-x^{\alpha_{1}} \alpha_{1} \beta_{1}+2 \gamma_{1}\right) \Gamma\left[\frac{2 \gamma_{2}}{\alpha_{2}}\right]}{\beta_{2}^{\frac{\gamma_{2}}{\alpha_{2}}} x^{\gamma_{2}} \Gamma\left[\frac{\gamma_{2}}{\alpha_{2}}\right]+\beta_{2} \Gamma\left[\frac{2 \gamma_{2}}{\alpha_{2}}\right]} \\
& \left.-\frac{\beta_{1} \gamma_{1} \Gamma\left[\frac{2 \gamma_{1}}{\alpha_{1}}\right]}{x^{\gamma_{1}} \beta_{1}^{\frac{\gamma_{1}}{\alpha_{1}}} \Gamma\left[\frac{\gamma_{1}}{\alpha_{1}}\right]+\beta_{1} \Gamma\left[\frac{2 \gamma_{1}}{\alpha_{1}}\right]}\right) .
\end{aligned}
$$

Clearly, if $\alpha_{1}=\alpha_{2}$ and $\beta_{1}=\beta_{2}$, then (8) is negative when $\gamma_{2}>2 \gamma_{1}$.

Lemma 2.2 Let $X_{1}$ and $X_{2}$ be two random variables having the GPL distribution with parameters vector $\left(\alpha_{i}, \beta_{i}, \gamma_{i}\right), i=1$, 2. If $\alpha_{1}=\alpha_{2}, \beta_{1}=\beta_{2}$ and $\gamma_{2}>2 \gamma_{1}$, then $X_{1} \pi_{l r} X_{2}$, $X_{1} \pi_{h r} X_{2}$ and $X_{1} \pi_{s} X_{2}$.

\section{Moments and associated measures}

The $r$ th moment of the GPL distribution is given by

$$
=\frac{\mu_{r}^{\prime}=E\left[X^{r}\right]}{\beta^{-\frac{r}{\alpha}}\left(\beta \Gamma\left[\frac{2 \gamma}{\alpha}\right] \Gamma\left[\frac{r+\gamma}{\alpha}\right]+\Gamma\left[\frac{\gamma}{\alpha}\right] \Gamma\left[\frac{r+2 \gamma}{\alpha}\right]\right)}, \quad \gamma+r>0 .
$$


Note that when $\gamma=\alpha=1$, i.e., in the case of Lindley distribution, the above expression simply reduces to

$$
\mu_{r}^{\square}=\frac{\beta^{-r}(1+r+\beta) \Gamma[1+r]}{1+\beta}, \quad r>-1,
$$

and when $\gamma=\alpha$, i.e., in the case of power Lindley distribution, the above expression simply reduces to

$$
\mu_{r}^{\square}=\frac{\beta^{-\frac{r}{\alpha}}(r+\alpha+\alpha \beta) \Gamma\left[\frac{r+\alpha}{\alpha}\right]}{\alpha(1+\beta)}, \alpha+r>0 .
$$

Therefore using (9), the mean and variance of the generalized power Lindley distribution, respectively, are

$$
\mu=E[X]=\frac{\beta^{-1 / \alpha}\left(\frac{\beta \Gamma\left[\frac{1+\gamma}{\alpha}\right]}{\Gamma\left[\frac{\gamma}{\alpha}\right]}+\frac{\Gamma\left[\frac{1+2 \gamma}{\alpha}\right]}{\Gamma\left[\frac{2 \gamma}{\alpha}\right]}\right)}{1+\beta},
$$

and

$$
\sigma^{2}=\frac{\beta^{-2 / \alpha}\left((1+\beta)\left(\frac{\Gamma\left[\frac{2(1+\gamma)}{\alpha}\right]}{\Gamma\left[\frac{2 \gamma}{\alpha}\right]}+\frac{\beta \Gamma\left[\frac{2+\gamma}{\alpha}\right]}{\Gamma\left[\frac{\gamma}{\alpha}\right]}\right)-\left(\frac{\beta \Gamma\left[\frac{1+\gamma}{\alpha}\right]}{\Gamma\left[\frac{\gamma}{\alpha}\right]}+\frac{\Gamma\left[\frac{1+2 \gamma}{\alpha}\right]}{\Gamma\left[\frac{2 \gamma}{\alpha}\right]}\right)^{2}\right)}{(1+\beta)^{2}} .
$$

The skewness and kurtosis measures can be obtained from the expressions

$$
\begin{gathered}
\text { Skewness }=\frac{\mu_{3}^{\prime \square}-3 \mu_{2}^{\prime} \mu+2 \mu^{3}}{\sigma^{3}}, \\
\text { Kurtosis }=\frac{\mu_{4}^{\prime}-4 \mu_{3}^{\prime} \mu+6 \mu_{2}^{\prime} \mu^{2}}{\sigma^{4}},
\end{gathered}
$$

upon substituting for the raw moments. Table 1 gives some properties of the GPL distribution for some values of the parameters.

Table 1: Moments of the GPL distribution for some parameter values.

\begin{tabular}{|c|c|c|c|c|c|c|c|c|}
\hline & \multicolumn{3}{|c|}{$\alpha=0.5, \beta=16$} & \multicolumn{3}{c|}{$\alpha=1, \beta=7$} & \multicolumn{2}{c|}{$\alpha=0.3, \beta=6$} \\
\hline & $\gamma=2.7$ & $\gamma=8.9$ & $\gamma=22$ & $\gamma=0.75$ & $\gamma=1.3$ & $\gamma=22$ & $\gamma=4$ & $\gamma=8$ \\
\hline$\mu$ & 0.156 & 1.531 & 0.907 & 0.12 & 0.209 & 3.536 & 39.867 & 365.723 \\
\hline$\sigma^{2}$ & 0.027 & 1.337 & 36.622 & 0.018 & 0.034 & 1.585 & 4771.08 & 326131 \\
\hline Skewness & 3.209 & 3.099 & 3.353 & 2.214 & 1.730 & 1.582 & 4.139 & 3.124 \\
\hline Kurtosis & -18.628 & -29.97 & -38.695 & 27.432 & -2.981 & -127.211 & -11.665 & -10.631 \\
\hline
\end{tabular}




\subsection{Characteristic function}

In this subsection, the characteristic function of the GPL distribution is derived. We know that

$$
\psi(t)=E[\exp \{i t X\}]=\sum_{n=0}^{\infty} \frac{(i t)^{n}}{n !} E\left[X^{n}\right] \quad t \in R .
$$

Hence using (9), the characteristic function of the GPL distribution is given by

$$
\phi(t)=\sum_{n=0}^{\infty} \frac{(i t)^{n} \beta^{-n / \alpha}\left(\beta \Gamma\left[\frac{2 \gamma}{\alpha}\right] \Gamma\left[\frac{n+\gamma}{\alpha}\right]+\Gamma\left[\frac{\gamma}{\alpha}\right] \Gamma\left[\frac{n+2 \gamma}{\alpha}\right]\right)}{n !(1+\beta) \Gamma\left[\frac{\gamma}{\alpha}\right] \Gamma\left[\frac{2 \gamma}{\alpha}\right]}, \gamma+n>0,
$$

where $i=\sqrt{-1}$ is the imaginary number.

\section{Mean residual life function}

In this section, the mean residual life function of the GPL distribution is given. Another important representation for a random variable is the mean residual life (MRL) function defined by

$$
m(t)=E[X-t \mid X>t]=\frac{\int_{t}^{\infty} x f(x) d x}{\bar{F}(t)}-t=\frac{\int_{t}^{\infty} \bar{F}(x) d x}{\bar{F}(t)},
$$

where $\bar{F}(x)=1-F(x)$ is the survival function.

The MRL function as well as failure rate function is very important since each of them can determine a unique corresponding life time distribution.

Lemma 4.1 The MRL function of the GPL distribution is

$$
m(t)=-t+\frac{t^{\gamma+1} \beta^{\frac{\gamma}{\alpha}}\left(t^{\gamma} \beta^{\frac{\gamma}{\alpha}} \mathrm{E} E\left[\frac{-1+\alpha-2 \gamma}{\alpha}, t^{\alpha} \beta\right] \Gamma\left[\frac{\gamma}{\alpha}\right]+t \beta \mathrm{E} E\left[\frac{-1+\alpha-\gamma}{\alpha}, t^{\alpha} \beta\right] \Gamma\left[\frac{2 \gamma}{\alpha}\right]\right)}{\beta \Gamma\left[\frac{2 \gamma}{\alpha}\right] \Gamma\left[\frac{\gamma}{\alpha}, t^{\alpha} \beta\right]+\Gamma\left[\frac{\gamma}{\alpha}\right] \Gamma\left[\frac{2 \gamma}{\alpha}, t^{\alpha} \beta\right]}, t>0,
$$

in which

$$
\mathrm{E} E(n, z)=\operatorname{ExplntegralE}(n, z)=E_{n}(z)=\int_{1}^{\infty} \frac{e^{-z t}}{t^{n}} d t .
$$

Proof. We have

$$
f(x ; \alpha, \beta, \gamma)=\frac{\beta}{\beta+1} f_{g g}(x ; \alpha, \beta, \gamma)+\frac{1}{\beta+1} f_{g g}(x ; \alpha, \beta, 2 \gamma),
$$

and thus with some elementary algebraic calculations, the proof is completed.

\section{Lorenz and Bonferroni curves}

In this section, we give the Lorenz and Bonferroni curves for our proposed distribution. 


\subsection{Lorenz curve}

The Lorenz curve for a positive random variable $X$ is defined as the graph of the ratio

$$
L_{F}(F(y))=\frac{1}{\mu} \int_{0}^{y} u f(u) d u
$$

against $F(x)$ with the properties $L(p)=p, L(0)=0$ and $L(1)=1$. If $X$ represents annual income, $L(p)$ is the proportion of total income that accrues to individuals having the $100 p \%$ lowest incomes.

If all individuals earn the same income then $L(p)=p$ for all $p$. The area between the line $L(p)=p$ and the Lorenz curve may be regarded as a measure of inequality of income, or more generally, of the variability of $X$, see Gail and Gastwirth [(1978)] and Dagum [(1985)] for extensive discussion of Lorenz curves.

Lemma 5.1 The Lorenze curve of GPL distribution is given by

$$
L_{F}(F(y))=\frac{\frac{\beta\left(\Gamma\left[\frac{1+\gamma}{\alpha}\right]-y^{1+\gamma}\left(y^{\alpha}\right)^{-\frac{1+\gamma}{\alpha}} \Gamma\left[\frac{1+\gamma}{\alpha}, y^{\alpha} \beta\right]\right)}{\Gamma\left[\frac{\gamma}{\alpha}\right]}+\frac{\Gamma\left[\frac{1+2 \gamma}{\alpha}\right]-y^{1+2 \gamma}\left(y^{\alpha}\right)^{-\frac{1+2 \gamma}{\alpha}} \Gamma\left[\frac{1+2 \gamma}{\alpha}, y^{\alpha} \beta\right]}{\Gamma\left[\frac{2 \gamma}{\alpha}\right]}}{\frac{\beta \Gamma\left[\frac{1+\gamma}{\alpha}\right]}{\Gamma\left[\frac{\gamma}{\alpha}\right]}+\frac{\Gamma\left[\frac{1+2 \gamma}{\alpha}\right]}{\Gamma\left[\frac{2 \gamma}{\alpha}\right]}} .
$$

Proof. We have

$$
f(x ; \alpha, \beta, \gamma)=\frac{\beta}{\beta+1} f_{g g}(x ; \alpha, \beta, \gamma)+\frac{1}{\beta+1} f_{g g}(x ; \alpha, \beta, 2 \gamma),
$$

and

$$
\mu=\frac{\beta^{-1 / \alpha}\left(\frac{\beta \Gamma\left[\frac{1+\gamma}{\alpha}\right]}{\Gamma\left[\frac{\gamma}{\alpha}\right]}+\frac{\Gamma\left[\frac{1+2 \gamma}{\alpha}\right]}{\Gamma\left[\frac{2 \gamma}{\alpha}\right]}\right)}{1+\beta} .
$$

The rest of the proof is straightforward.

\subsection{Bonferroni curve}

The Bonferroni curve has many applications not only in Economics to study income and poverty, but also in other fields like reliability, medicine and insurance. The Bonferroni curve $B_{F}[F(y)]$ is given by

$$
B_{F}[F(y)]=\frac{1}{\mu F(y)} \int_{0}^{y} u f(u) d u \text {. }
$$


Therefore the Bonferroni curve of $F$ that follows the GPL distribution can be obtained via the expression $B_{F}[F(y)]=L_{F}(F(y)) / F(y)$, where

$$
F(y)=1-\frac{\frac{\beta \Gamma\left[\frac{\gamma}{\alpha}, x^{\alpha} \beta\right]}{\Gamma\left[\frac{\gamma}{\alpha}\right]}+\frac{\Gamma\left[\frac{2 \gamma}{\alpha}, x^{\alpha} \beta\right]}{\Gamma\left[\frac{2 \gamma}{\alpha}\right]} .}{1+\beta} .
$$

\section{Characterizations of GPL distribution}

In designing a stochastic model for a particular modeling problem, an investigator will be vitally interested to know if their model fits the requirements of a specific underlying probability distribution. To this end, the investigator will rely on the characterizations of the selected distribution. Generally speaking, the problem of characterizing a distribution is an important problem in various fields and has recently attracted the attention of many researchers. Consequently, various characterization results have been reported in the literature. These characterizations have been established in many different directions. The present section deals with the characterizations of GPL distribution. These characterizations are based on a simple relationship between two truncated moments. Our characterization results presented here will employ an interesting result due to Glänzel [(1987)] (Theorem 6.1 below). The advantage of the characterizations given here is that, cdf $F$ need not have a closed form and are given in terms of an integral whose integrand depends on the solution of a first order differential equation, which can serve as a bridge between probability and differential equation.

Theorem 6.1 Let $(\Omega, \Phi, P)$ be a given probability space and let $H=[a, b]$ be an interval for some $a<b \quad(a=-\infty, b=\infty$ might as well be allowed $)$. Let $X: \Omega \rightarrow H$ be a continuous random variable with the distribution function $F$ and let $g$ and $h$ be two real functions defined on $H$ such that

$$
\mathbf{E}[g(X) \mid X \geq x]=\mathbf{E}[h(X) \mid X \geq x] \eta(x), \quad x \in H,
$$

is defined with some real function $\eta$. Assume that $g h \in C^{1}(H), \eta \in C^{2}(H)$ and $F$ is twice continuously differentiable and strictly monotone function on the set $H$. Finally, assume that the equation $h \eta=g$ has no real solution in the interior of $H$. Then $F$ is uniquely determined by the functions $g, h$ and $\eta$, particularly

$$
F(x)=\int_{a}^{x} C\left|\frac{\eta^{\prime}(u)}{\eta(u) h(u)-g(u)}\right| \exp (-s(u)) d u,
$$

where the function $s$ is a solution of the differential equation $s^{\prime}=\frac{\eta^{\prime} h}{\eta h-g}$ and $C$ is a constant, chosen to make $\int_{H} d F=1$.

We like to mention that this kind of characterization based on the ratio of truncated moments is stable in the sense of weak convergence, in particular, let us assume that there is a sequence $\left\{X_{n}\right\}$ of random variables with distribution functions $\left\{F_{n}\right\}$ such that 
the functions $g_{n}, h_{n}$ and $\eta_{n}(n \in \mathrm{N})$ satisfy the conditions of Theorem 6.1 and let $g_{n} \rightarrow g, h_{n} \rightarrow h$ for some continuously differentiable real functions $g$ and $h$. Let, finally, $X$ be a random variable with distribution $F$. Under the condition that $g_{n}(X)$ and $h_{n}(X)$ are uniformly integrable and the family $\left\{F_{n}\right\}$ is relatively compact, the sequence $X_{n}$ converges to $X$ in distribution if and only if $\eta_{n}$ converges to $\eta$, where

$$
\eta(x)=\frac{E[g(X) \mid X \geq x]}{E[h(X) \mid X \geq x]}
$$

This stability theorem makes sure that the convergence of distribution functions is reflected by corresponding convergence of the functions $g, h$ and $\eta$, respectively.

Remark 6.2 $(a)$ In Theorem 6.1, the interval $H$ need not be closed since the condition is only on the interior of $H$. (b) Clearly, Theorem 6.1 can be stated in terms of two functions $g$ and $\eta$ by taking $h(x) \equiv 1$, provided that the $c d f \quad F$ has a closed form, which will reduce the condition given in Theorem 6.1 to $E[g(X) \mid X \geq x]=\eta(x)$. However, adding an extra function will give a lot more flexibility, as far as its application is concerned.

Proposition 6.3 Let $X: \Omega \rightarrow(0, \infty)$ be a continuous random variable and let $h(x)=$ $g(x) e^{-\beta x^{\alpha}}$ and $g(x)=\left(\beta \Gamma\left(\frac{2 \gamma}{\alpha}\right)+x^{\gamma} \beta^{\gamma / \alpha} \Gamma\left(\frac{\gamma}{\alpha}\right)\right)^{-1} x^{\alpha-\gamma}$ for $x \in(0, \infty)$. The pdf of $X$ is

(4) if and only if the function $\eta$ defined in Theorem 6.1 has the form

$$
\eta(x)=2 e^{\beta x^{\alpha}}, x>0 \text {. }
$$

Proof. Let $X$ have density (4), then

$$
(1-F(x)) \mathbf{E}[h(X) \mid X \geq x]=\frac{\beta^{\frac{\gamma}{\alpha}-1} e^{-2 \beta x^{\alpha}}}{2(1+\beta) \Gamma\left(\frac{\gamma}{\alpha}\right) \Gamma\left(\frac{2 \gamma}{\alpha}\right)}, \quad x>0,
$$

and

$$
(1-F(x)) \mathbf{E}[g(X) \mid X \geq x]=\frac{\beta^{\frac{\gamma}{\alpha}-1} e^{-\beta x^{\alpha}}}{(1+\beta) \Gamma\left(\frac{\gamma}{\alpha}\right) \Gamma\left(\frac{2 \gamma}{\alpha}\right)}, \quad x>0,
$$

and finally

$$
\eta(x) h(x)-g(x)=g(x)>0, \quad x>0 .
$$

Conversely, if $\eta$ is given as above, then

$$
s^{\prime}(x)=\frac{\eta^{\prime}(x) h(x)}{\eta(x) h(x)-g(x)}=2 \alpha \beta x^{\alpha-1}, \quad x>0,
$$

and hence 
A generalized power Lindley distribution: Model, Properties and Applications.

$$
s(x)=2 \beta x^{\alpha}, \quad x>0 .
$$

Now, in view of Theorem 6.1, $X$ has density (4).

Corollary 6.4 Let $X: \Omega \rightarrow(0, \infty)$ be a continuous random variable and let $h(x)$ be as in Proposition 6.3. The $p d f$ of $X$ is (4) if and only if there exist functions $g$ and $\eta$ defined in Theorem 6.1 satisfying the differential equation

$$
\frac{\eta^{\prime}(x) h(x)}{\eta(x) h(x)-g(x)}=2 \alpha \beta x^{\alpha-1}, x>0 \text {. }
$$

\section{Remark 6.5}

(a) The general solution of the differential equation in Corollary 6.4 is

$$
\eta(x)=e^{2 \beta x^{\alpha}}\left\lfloor-\int 2 \alpha \beta x^{\alpha-1} e^{-2 \beta x^{\alpha}}(h(x))^{-1} g(x) d x+D\right\rfloor
$$

for $x>0$, where $D$ is a constant. One set of appropriate functions is given in Proposition 6.3 with $D=0$.

(b) Clearly there are other triplets of functions $(h, g, \eta)$ satisfying the conditions of

Theorem 6.1. We presented one such triplet in Proposition 6.3.

\section{Different methods for estimating}

In this section, the maximum likelihood and the minimum spacing distance estimators are discussed and compared.

\subsection{Maximum likelihood estimation}

In this subsection, the maximum likelihood estimators of $\operatorname{GPL}(\alpha, \beta, \gamma)$ are considered. If $X_{1}, \ldots \ldots X_{n}$ is a random sample from the GPL distribution, then the log-likelihood function, $l(\alpha, \beta, \gamma)$ is:

$$
\begin{aligned}
& l(\alpha, \beta, \gamma)=-\beta \sum_{i=1}^{\infty} x_{i}^{\alpha}+(\gamma-1) \sum_{i=1}^{\infty} \log \left(x_{i}\right) \\
& +n \log \left(\alpha \beta^{\frac{\gamma}{\alpha}}\right)+\sum_{i=1}^{\infty} \log \left(x_{i}^{\gamma} \beta^{\frac{\gamma}{\alpha}} \Gamma\left[\frac{\gamma}{\alpha}\right]+\beta \Gamma\left[\frac{2 \gamma}{\alpha}\right]\right) \\
& -n \log \left((1+\beta) \Gamma\left[\frac{\gamma}{\alpha}\right] \Gamma\left[\frac{2 \gamma}{\alpha}\right]\right) .
\end{aligned}
$$

Therefore, the normal equations are

$$
\frac{\partial l}{\partial \alpha}=\frac{1}{\alpha^{2}}\left(n\left(\alpha-\gamma \log (\beta)+\gamma \mathrm{P} G\left[0, \frac{\gamma}{\alpha}\right]+2 \gamma \mathrm{P} G\left[0, \frac{2 \gamma}{\alpha}\right]\right)\right.
$$




$$
\begin{aligned}
& \left.+\left(-\beta \alpha^{2} \sum_{i=1}^{\infty} \log \left(x_{i}\right) x_{i}^{\alpha}+\sum_{i=1}^{\infty} \frac{-2 \beta \gamma\left[\frac{2 \gamma}{\alpha}\right] \mathrm{P} G\left[0, \frac{2 \gamma}{\alpha}\right]-\beta^{\frac{\gamma}{\alpha}} \gamma\left[\frac{\gamma}{\alpha}\right] \log (\beta) x_{i}^{\gamma}-\beta^{\frac{\gamma}{\alpha}} \gamma\left[\frac{\gamma}{\alpha}\right] \mathrm{P} G\left[0, \frac{\gamma}{\alpha}\right] x_{i}^{\gamma}}{\beta \Gamma\left[\frac{2 \gamma}{\alpha}\right]+\beta^{\frac{\gamma}{\alpha}} \Gamma\left[\frac{\gamma}{\alpha}\right] x_{i}^{\gamma}}\right)\right) \\
& =0, \\
& \frac{\partial l}{\partial \beta}=-\frac{n}{1+\beta}+\frac{n \gamma}{\alpha \beta}-\sum_{i=1}^{\infty} x_{i}^{\alpha}+\sum_{i=1}^{\infty} \frac{\Gamma\left[\frac{2 \gamma}{\alpha}\right]+\beta^{-1+\frac{\gamma}{\alpha}} \Gamma\left[1+\frac{\gamma}{\alpha}\right] x_{i}^{\gamma}}{\beta \Gamma\left[\frac{2 \gamma}{\alpha}\right]+\beta^{\frac{\gamma}{\alpha}} \Gamma\left[\frac{\gamma}{\alpha}\right] x_{i}^{\gamma}}=0 \\
& \frac{\partial l}{\partial \gamma}=\frac{1}{\alpha}\left(n\left(\log (\beta)-\mathrm{P} G\left[0, \frac{\gamma}{\alpha}\right]-2 \mathrm{P} G\left[0, \frac{2 \gamma}{\alpha}\right]\right)\right. \\
& +\alpha \sum_{i=1}^{\infty} \log \left(x_{i}\right) \\
& \left.+\sum_{i=1}^{\infty} \frac{2 \beta \Gamma\left[\frac{2 \gamma}{\alpha}\right] \mathrm{P} G\left[0, \frac{2 \gamma}{\alpha}\right]+\beta^{\frac{\gamma}{\alpha}} \Gamma\left[\frac{\gamma}{\alpha}\right] \log (\beta) x_{i}^{\gamma}+\alpha \beta^{\frac{\gamma}{\alpha}} \Gamma\left[\frac{\gamma}{\alpha}\right] \log \left(x_{i}\right) x_{i}^{\gamma}+\beta^{\frac{\gamma}{\alpha}} \Gamma\left[\frac{\gamma}{\alpha}\right] \mathrm{P} G\left[0, \frac{\gamma}{\alpha}\right] x_{i}^{\gamma}}{\beta \Gamma\left[\frac{2 \gamma}{\alpha}\right]+\beta^{\frac{\gamma}{\alpha}} \Gamma\left[\frac{\gamma}{\alpha}\right] x_{i}^{\gamma}}\right) \\
& =0,
\end{aligned}
$$

where

$$
\mathrm{P} G[n, z]=\operatorname{PolyGamma}[n, z]=\psi^{(n)}(z)=\frac{\partial^{n} \psi(z)}{\partial z^{n}},
$$

and

$$
\psi(z)=\frac{\Gamma^{\prime}(z)}{\Gamma(z)},
$$

The maximum likelihood estimates $\hat{\alpha}, \hat{\beta}$ and $\hat{\gamma}$ for the parameters $\alpha, \beta, \gamma$, respectively, can be obtained by solving iteratively Equations (11)-(13).

\subsection{Minimum spacing distance estimator}

In this subsection, we provide the minimum spacing distance estimator (MSDE) of the generalized power Lindley distribution. Let $X_{1}, \mathrm{~K}, X_{n}$ be a random sample from continuous function $F_{\theta}, \boldsymbol{\theta} \in \boldsymbol{\Theta} \subset R^{k}$ with support on $R$. Let the order statistics be denoted by $Y_{1}, \mathrm{~K}, Y_{n}$. Define

$$
D_{i}(\boldsymbol{\theta})=F_{\boldsymbol{\theta}}\left(Y_{i}\right)-F_{\boldsymbol{\theta}}\left(Y_{i-1}\right), \quad i=1, \mathrm{~K}, n+1,
$$

where $F_{\boldsymbol{\theta}}\left(Y_{0}\right)=0$ and $F_{\boldsymbol{\theta}}\left(Y_{n+1}\right)=1$. The MSDE of $\boldsymbol{\theta}$ is obtained the estimators by minimizing

$$
T(\boldsymbol{\theta})=\sum_{i=1}^{n+1} h\left(D_{i}(\boldsymbol{\theta}), \frac{1}{n+1}\right),
$$

in which $h(x, y)$ is an appropriate distance. Some choices of $h(x, y)$ are $|x-y|$ and $|\ln x-\ln y|$, which are called "absolute" and "absolute-log" distance, respectively. The 
A generalized power Lindley distribution: Model, Properties and Applications.

corresponding estimators are called "minimum spacing absolute distance estimator" (MSADE) and "minimum spacing absolute-log distance estimator" (MSALDE). This method was originally explored by Torabi [(2008)] and it is used quite successfully for the generalized power Lindley distribution.

\section{Simulation method for the GPL distribution}

The density function of the GPL distribution can be written in terms of the generalized gamma density function as

$$
f(x ; \alpha, \beta, \gamma)=\frac{\beta}{\beta+1} f_{g g}(x ; \alpha, \beta, \gamma)+\frac{1}{\beta+1} f_{g g}(x ; \alpha, \beta, 2 \gamma),
$$

To generate random data $X_{i}, i=1, \ldots, n$, from $\operatorname{GPL}(\alpha, \beta, \gamma)$, one can use the following algorithm:

1. Generate $U_{i}, i=1, \ldots, n$, from $U(0,1)$ distribution.

2. Generate $V_{1 i}, i=1, \ldots, n$, from the $\operatorname{Stacy} \operatorname{gamma}(\alpha, \beta, \gamma)$.

3. Generate $V_{2 i}, i=1, \ldots, n$, from the $\operatorname{Stacy} \operatorname{gamma}(\alpha, \beta, 2 \gamma)$.

4. If $U_{i} \leq \frac{\beta}{\beta+1}$, then set $X i=V_{1 i}$; otherwise set $X_{i}=V_{2 i}, i=1, \ldots, n$.

\section{Simulation study}

We simulate $n=20,30,50,100$ and 200 times the generalized power Lindley distribution for $\alpha=0.2, \beta=4$ and $\gamma=0.5$. For each sample size, we compute the MLE's, MME's, MSADE's and MSALDE's of the parameters. We repeat this process 1000 times and compute the average estimate (AE) and MSE. The results are reported in Table 2.

Table 2: Estimated AE and MSE of MLE, MME and MSLDE of parameters based on 1000 simulations of the generalized power Lindley distribution for $\alpha=0.2, \beta=4$ and $\gamma=0.5$ and with $n=20,30,50,100$ and 200.

\begin{tabular}{|c|c|c|c|c|c|c|c|c|c|}
\hline & \multicolumn{2}{|c|}{ MLE } & \multicolumn{2}{c|}{ MME } & \multicolumn{2}{c|}{ MSADE } & \multicolumn{2}{c|}{ MSALDE } \\
\hline$n$ & & AE & MSE & AE & MSE & AE & MSE & AE & MSE \\
\hline \multirow{4}{*}{20} & $\alpha$ & 0.37 & 0.794 & 1.082 & 0.898 & 0.230 & 0.035 & 0.242 & 0.082 \\
\cline { 2 - 11 } & $\beta$ & 5.306 & 25.960 & 0.379 & 13.667 & 3.924 & 1.294 & 4.785 & 19.331 \\
\cline { 2 - 11 } & $\gamma$ & 0.596 & 0.123 & 5.248 & 302.489 & 0.480 & 0.019 & 0.513 & 0.079 \\
\hline \multirow{3}{*}{30} & $\alpha$ & 0.281 & 0.156 & 0.997 & 0.736 & 0.212 & 0.012 & 0.214 & 0.022 \\
\cline { 2 - 11 } & $\beta$ & 5.114 & 17.336 & 0.378 & 13.459 & 3.972 & 1.345 & 4.660 & 14.359 \\
\cline { 2 - 11 } & $\gamma$ & 0.578 & 0.095 & 6.181 & 352.755 & 0.481 & 0.016 & 0.507 & 0.053 \\
\hline \multirow{3}{*}{50} & $\alpha$ & 0.230 & 0.016 & 0.946 & 0.660 & 0.211 & 0.010 & 0.211 & 0.011 \\
\cline { 2 - 11 } & $\beta$ & 4.504 & 7.508 & 0.427 & 13.538 & 4.011 & 1.521 & 4.398 & 8.675 \\
\cline { 2 - 11 } & $\gamma$ & 0.535 & 0.046 & 7.509 & 468.317 & 0.487 & 0.014 & 0.501 & 0.038 \\
\hline \multirow{4}{*}{100} & $\alpha$ & 0.207 & 0.002 & 0.883 & 0.588 & 0.205 & 0.002 & 0.203 & 0.003 \\
\cline { 2 - 10 } & $\beta$ & 4.381 & 3.144 & 0.553 & 12.527 & 4.007 & 1.185 & 4.255 & 3.604 \\
\cline { 2 - 10 } & $\gamma$ & 0.528 & 0.019 & 8.825 & 454.427 & 0.490 & 0.009 & 0.501 & 0.020 \\
\hline \multirow{3}{*}{200} & $\alpha$ & 0.204 & 0.001 & 0.878 & 0.609 & 0.202 & 0.001 & 0.201 & 0.001 \\
\cline { 2 - 10 } & $\beta$ & 4.167 & 1.514 & 0.672 & 12.122 & 4.023 & 0.812 & 4.101 & 1.390 \\
\cline { 2 - 10 } & $\gamma$ & 0.512 & 1.010 & 11.675 & 612.257 & 0.497 & 0.006 & 0.499 & 0.009 \\
\hline
\end{tabular}


Comparing the performance of all the estimators, it is observed that for all methods, the MSE's decrease as the sample size increases. Note that, the performances of the MSADE's are the best as far as the MSE is concerned, but after this method, the MLE's and the MME's performances are considerable. Considering all the points, we recommend to use the MSADE for estimating of parameters.

\section{Applications}

In this section, we use three real data sets to show that the generalized power Lindley distribution can be a better model than the power Lindley and Lindley distributions. In order to compare the models, estimates of the parameters of the distributions, Akaike Information Criterion $(\mathrm{A} I C=-2 \log \hat{L}+2 k)$, Bayesian Information Criterion $(\mathrm{B} I C=-2 \log \hat{L}+k \log n), \quad$ Consistent Akaike Information Criterion $\left(\mathrm{CAIC}=\mathrm{AIC}+\frac{2 k(k+1)}{n-k 1}\right) \quad$ and Hannan-Quinn information criterion ( HQIC $=-2 \log \hat{L}+2 \log (\log (n)) k$, where $\hat{L}$ is the value of the likelihood function evaluated at the parameter estimates, $n$ is the number of observations and $k$ is the number of estimated parameters. For fitting a data set, the best model is a model with the smallest value of AIC, BIC, CAIC and HQIC. We can also perform formal goodness-offit tests in order to verify which distribution fits better to these data. We apply Kolmogorov-Smirnov (KS), Anderson-Darling (AD) and Cramer Von Mises (CVM) statistics, where small values of theses statistics for models indicate that these models could be chosen as the best model to fit the data. These statistics evaluations were implemented using the $\mathrm{R}$ software through the commands ks.test, ad.test and cvm.test (for the last two commands, the package nortest is required).

The first data set represents the maintenance data with 52 observations reported on data concerning the Oits IQ Scores for 52 non-White males hired by a large insurance company in 1971, Roberts [(1988)]. It consists of the observations listed below:

$$
\begin{aligned}
& 91,102,100,117,122,115,97,109,108,104,108,118,103, \\
& 123,123,103,106,102,118,100,103,107,108,107,97,95, \\
& 119,102,108,103,102,112,99,116,114,102,111,104,122, \\
& 103,111,101,91,99,121,97,109,106,102,104,107,95 .
\end{aligned}
$$

The second data set represents the number of successive failures for the air conditioning system of each member in a fleet of 13 Boeing 720 jet airplanes reported in Proschan [(1963)]. See the listed below:

$194,413,90,74,55,23,97,50,359,50,130,487,57,102,15,14,10,57,320,261,51,44,9,254,33$, $18,209,41,58,60,48,56,87,11,102,12,5,14,14,29,37,186,29,104,7,4,72,270,283,7,493,61$, $100,61,502,220,120,141,22,603,35,98,54,100,11,181,65,49,12,239,14,18,39,3,12,5,32,9$, $438,43,134,184,20,386,182,71,80,188,230,152,5,36,79,59,33,246,1,79,3,27,201,84,27,156$, $21,16,88,130,14,118,44,15,42,106,46,230,26,59,153,104,20,206,5,66,34,29,26,35,5,82$, $31,118,326,12,54,36,34,18,25,120,31,22,18,216,139,67,310,3,46,210,57,76,14,111,97,62$, $39,30,7,44,11,63,23,22,23,14,18,13,34,16,18,130,90,163,208,1,24,70,16,101,52,208$ $95,62,11,191,14,71$. 
A generalized power Lindley distribution: Model, Properties and Applications.

The third data set consists Kevlar 49/Epoxy Strands Failure at 0.7 Stress Level Andrews [(1985)]. The data is presented the listed below:

$1051,1337,1389,1921,1942,2322,3629,4006,4012,4063,4921,5445,5620,5817$, $5905,5956,6068,6121,6473,7501,7886,8108,8546,8666,8831,9106,9711$, $9806,10205,10396,10861,11026,11214,11362,11604,11608,11745,11762$, $11895,12044,13520,13670,14110,14496,15395,16179,17092,17568,17568$.

Estimates of the parameters of GPL distribution, AICs, BICs, CAICs, HQICs, Kolmogorov-Smirnov, Anderson-Darling and Cramer Von Mises statistics are given in Tables 5, 6 and 7 for data sets 1-3, respectively. From these tables, we conclude that the GPL distribution provides a better fit to this data than the PL and Lindley distributions.

The plots of the empirical and theoretical density and cumulative distribution function (cdf) (left plots) and Q-Q and P-P plots (Right plots) are given in Figures 3-5. These figures show again that the GPL distribution gives a good fit for these data.

Table 3: MLEs, KS, AD and CVM statistics, AIC, BIC, CAIC, HQIC for the first real data set.

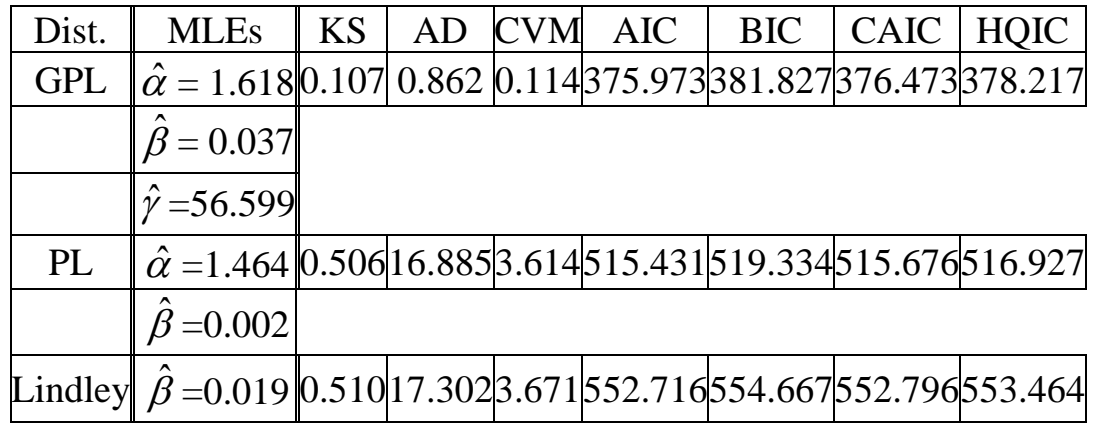

Figure 3: Fitted plots for the first real data set.
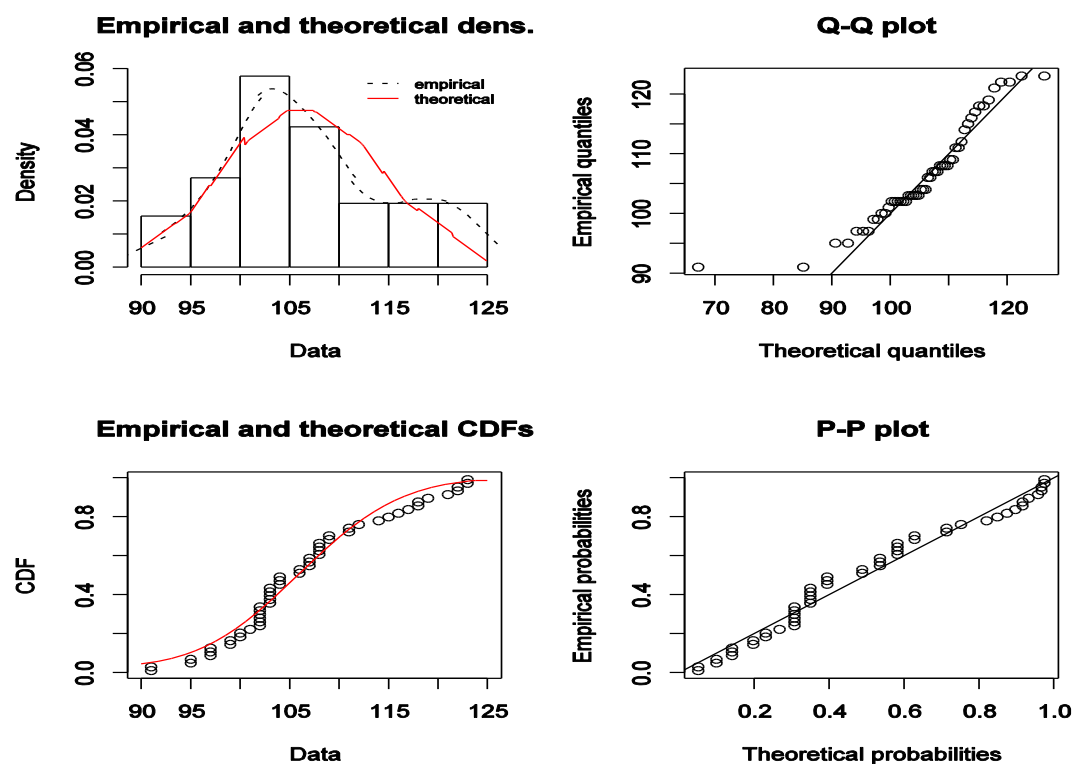
Table 4: MLEs, KS, AD and CVM statistics, AIC, BIC, CAIC, HQIC for the second real data set.

\begin{tabular}{|c|c|c|c|c|c|c|c|c|}
\hline Dist. & MLEs & $\mathrm{KS}$ & $\mathrm{AD}$ & CVM & AIC & $\mathrm{BIC}$ & CAIC & HQIC \\
\hline \multirow[t]{3}{*}{ GPL } & $\hat{\alpha}=0.411$ & 0.041 & 0.251 & 0.037 & 2070.888 & 2080.598 & 2071.018 & 2074.822 \\
\hline & $\hat{\beta}=1.582$ & & & & & & & \\
\hline & $\hat{\gamma}=2.625$ & & & & & & & \\
\hline \multirow[t]{2}{*}{ PL } & $\hat{\alpha}=0.660$ & 0.048 & 0.715 & \begin{tabular}{|l|}
0.108 \\
\end{tabular} & 2075.393 & 2081.865 & 2075.458 & 2078.01 \\
\hline & $\hat{\beta}=0.109$ & & & & & & & \\
\hline Lindley & $\hat{\beta}=0.022$ & 0.215 & 23.547 & 3.143 & 2167.309 & 2170.546 & 2167.331 & 2168.62 \\
\hline
\end{tabular}

Figure 4: Fitted plots for the second real data set.

\section{Empirical and theoretical dens.}

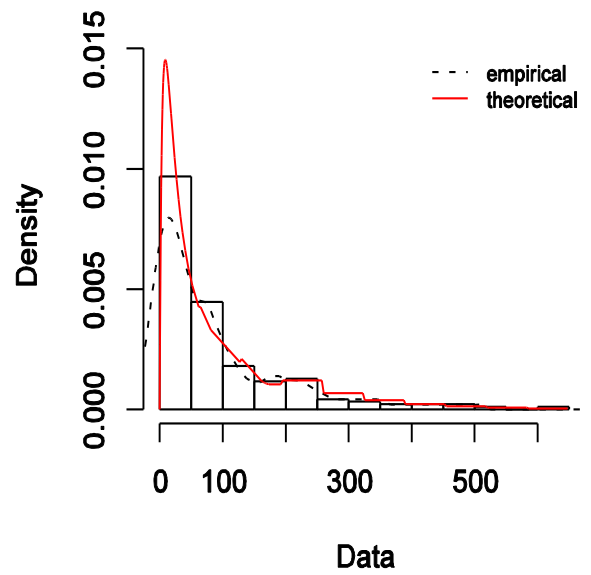

Empirical and theoretical CDFs

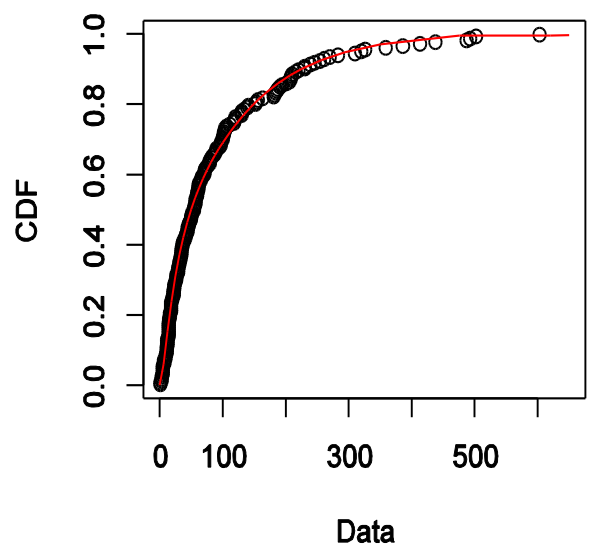

Q-Q plot

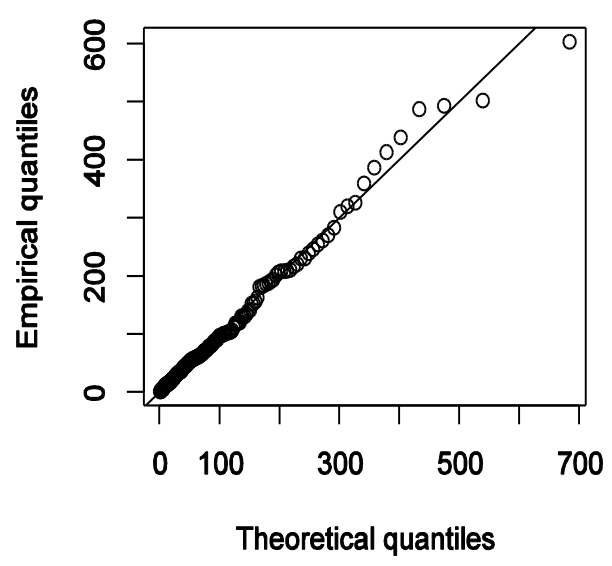

P.P plot

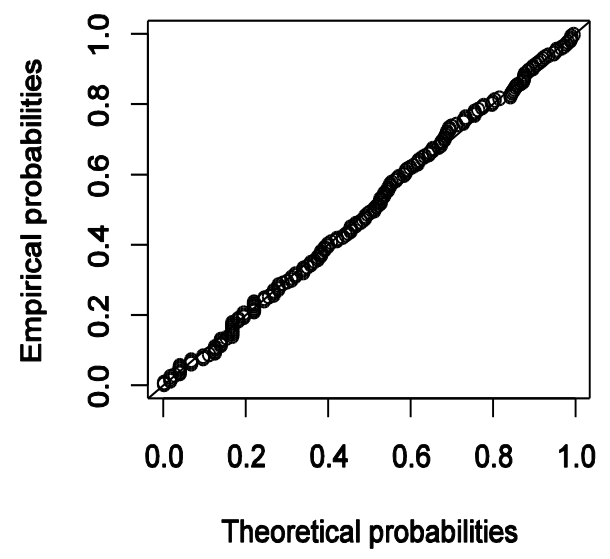


A generalized power Lindley distribution: Model, Properties and Applications.

Table 5: MLEs, KS, AD and CVM statistics, AIC, BIC, CAIC, HQIC for the third real data set.

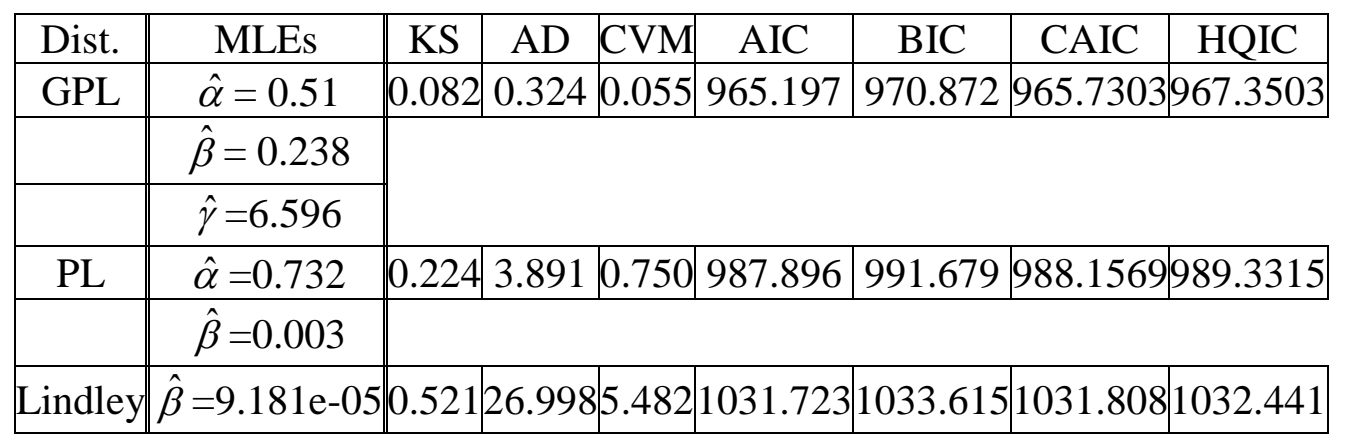

Figure 5: Fitted plots for the third real data set.

Empirical and theoretical dens.

Q-Q plot
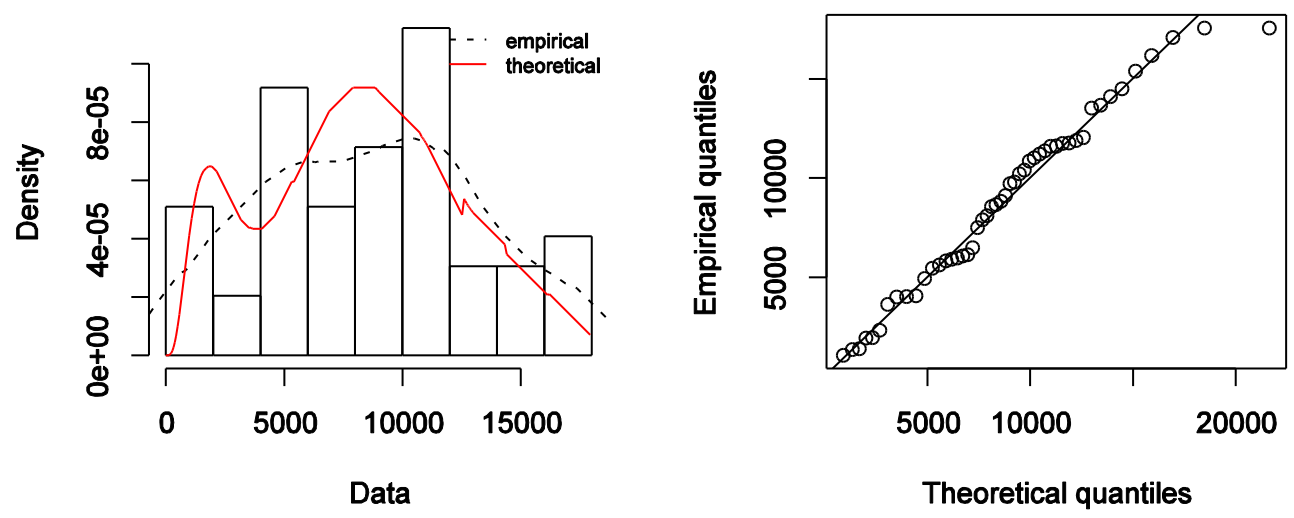

Empirical and theoretical CDFs
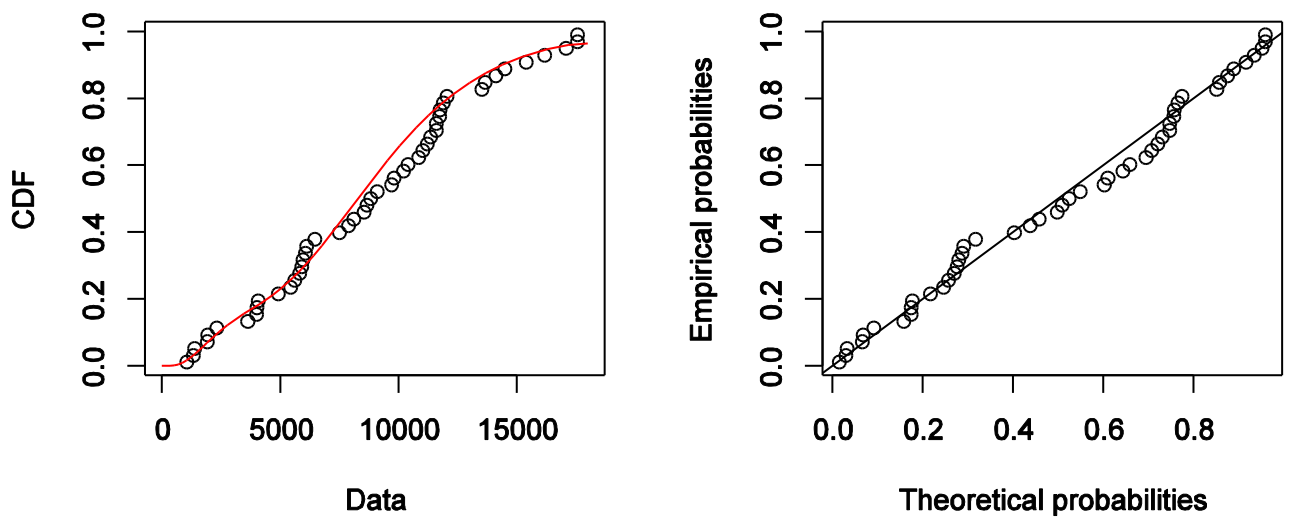


\section{Concluding remarks}

In this article, we introduced a new flexible generalization of the power Lindley distribution. We derived some important properties of the new distribution and application to three real data sets were presented and discussed to demonstratethat this distribution can be used quite effectively to provide better fit than other available subclass models such as the power Lindley and Lindley distributions.

\section{References}

1. Andrews, D. F. and Herzberg, A. M. (1985). Data: A Collection of Problems from Many Fields for the Student and Research Worker. New York: Springer Series in Statistics.

2. Dagum, C. (1985). Lorenz curve, in: S. Kotz, N.L. Johnson, C.B. Read (Eds.), in: Encyclopedia of Statistical Sciences, Vol. 5, Wiley, New York: 156-161.

3. Gail, M.H. and Gastwirth, J.L. (1978). A scale-free goodness-of-fit test for the exponential distribution based on the Lorenz curve, Journal of the American Statistical Association, 73, 787-793.

4. Ghitany, M.E., Al-Mutairi, D.K., Balakrishnan, N. and Al-Enezi, L.J. (2013). Power Lindley distribution and associated inference. Computational Statistics and Data Analysis, 64, 20-33.

5. Glänzel, W. (1987). A characterization theorem based on truncated moments and its application to some distribution families, Mathematical Statistics and Probability Theory (Bad Tatzmannsdorf, 1986), Vol. B, Reidel, Dordrecht, 75-84.

6. Lindley, D.V. (1958). Fiducial distributions and Bayes' theorem. Journal of the Royal Society, Series B, 20: 102-107.

7. Proschan, F. (1963). Theoretical explanation of observed decreasing failure rate. Technometrics, 5, 375-383.

8. Roberts, HV. (1988). Data Analysis for Managers with Minitab. Scientific Press, Redwood City, CA.

9. Shaked, M. and Shanthikumar, G. (1994). Stochastic Orders. Springer: New York.

10. Torabi, H. (2008). A general method for estimating and hypotheses testing using spacing. Journal of Statistical Theory and Applications, 8(2): 163-168. 